\title{
Erratum to: Induction of the Matrix Metalloproteinase 13 Gene in Bronchial Epithelial Cells by Interferon and Identification of its Novel Functional Polymorphism
}

\author{
Yoichi Mashimo, ${ }^{1}$ Mika Sakurai-Yageta, ${ }^{2}$ Misa Watanabe, ${ }^{3}$ Takayasu Arima, ${ }^{4}$ Yoshinori Morita, \\ Yuzaburo Inoue, ${ }^{4}$ Kazuki Sato, ${ }^{5}$ Toshiyuki Nishimuta, ${ }^{5}$ Shuichi Suzuki, ${ }^{5}$ Hiroko Watanabe, \\ Akira Hoshioka, ${ }^{6}$ Minako Tomiita, ${ }^{6}$ Akiko Yamaide, ${ }^{6}$ Yoichi Kohno, ${ }^{4}$ Yoshitaka Okamoto, ${ }^{7}$ \\ Naoki Shimojo, ${ }^{4}$ Akira Hata, ${ }^{1}$ and Yoichi Suzuki ${ }^{1,2,8}$
}

\section{Erratum to: Inflammation \\ DOI 10.1007/s10753-015-0291-1}

In the article published in Inflammation, with a DOI 10.1007/s10753-015-0291-1, the name of the second author was misspelled. The correct name is Mika Sakurai-Yageta. The authors regret this error.

The correct name is now captured above.

The online version of the original article can be found at http://dx.doi.org/ 10.1007/s10753-015-0291-1.

\footnotetext{
${ }^{1}$ Department of Public Health, Graduate School of Medicine, Chiba University, Chiba, Japan

${ }^{2}$ Department of Education and Training, Tohoku Medical Megabank Organization, Tohoku University, 2-1 Seiryomachi, Aobaku, Sendai, 980-8573, Japan

${ }^{3}$ The Department of Pediatrics, Toho University School of Medicine, Tokyo, Japan

${ }^{4}$ Department of Pediatrics, Graduate School of Medicine, Chiba University, Chiba, Japan

${ }^{5}$ Department of Pediatrics, National Shimoshizu Hospital, Yotsukaido, Japan

${ }^{6}$ Department of Allergy and Rheumatology, Chiba Children's Hospital, Chiba, Japan

${ }^{7}$ Department of Otorhinolaryngology and Head and Neck Surgery, Graduate School of Medicine, Chiba University, Chiba, Japan

${ }^{8}$ To whom correspondence should be addressed at Department of Education and Training, Tohoku Medical Megabank Organization, Tohoku University, 2-1 Seiryomachi, Aobaku, Sendai, 980-8573, Japan. E-mail: ysuzuki@megabank.tohoku.ac.jp
} 